\title{
AstroBiology Explorer (ABE) MIDEX mission concept: identifying organic molecules in space
}

Kimberly A. Ennico, Scott Sandford, Louis Allamandola, Jesse D. Bregman, Martin Cohen, et al.

Kimberly A. Ennico, Scott Sandford, Louis Allamandola, Jesse D. Bregman, Martin Cohen, Dale Cruikshank, Thomas P. Greene, Douglas Hudgins, Sun Kwok, Steven D. Lord, Suzanne Madden, Craig R. McCreight, Thomas L. Roellig, Donald W. Strecker, A. G. G. M. Tielens, Michael W. Werner, "AstroBiology Explorer (ABE) MIDEX mission concept: identifying organic molecules in space," Proc. SPIE 4850, IR Space Telescopes and Instruments, (5 March 2003); doi: 10.1117/12.461145

Event: Astronomical Telescopes and Instrumentation, 2002, Waikoloa, Hawai'i, United States 


\title{
The AstroBiology Explorer (ABE) MIDEX Mission Concept - Identifying Organic Molecules in Space
}

\author{
K. Ennico, ${ }^{a}$ S. Sandford, ${ }^{a}$ L. Allamandola, ${ }^{a}$ J. Bregman, ${ }^{a}$ M. Cohen, ${ }^{b}$ D. Cruikshank, ${ }^{a}$ \\ T. Greene, ${ }^{a}$ D. Hudgins, ${ }^{a}$ S. Kwok, ${ }^{c}$ S. Lord, ${ }^{d}$ S. Madden,,${ }^{e}$ C. McCreight, ${ }^{a}$ T. Roellig, ${ }^{a}$ \\ D. Strecker, ${ }^{\mathrm{f}}$ A. Tielens, ${ }^{\mathrm{g}}$ M. Werner ${ }^{\mathrm{h}}$ \\ ${ }^{a}$ NASA-Ames Research Center, Moffett Field, CA, 94035 USA \\ ${ }^{\mathrm{b}}$ Radio Astronomy Laboratory, 601 Campbell Hall, Univ. of California, Berkeley, CA 94720 \\ ${ }^{c}$ Dept. Physics \& Astronomy, Univ. of Calgary, Calgary, Alberta, Canada T2N 1N4 \\ ${ }^{\mathrm{d}}$ IPAC/Caltech, MS 100-22, Pasadena, CA 91125 \\ e'Service d'Astrophysique, CEA, Saclay, Orme des Merisiers, Bat. 709, F-91191, France \\ ${ }^{\mathrm{f}}$ Ball Aerospace, P.O. Box 1062, Boulder, CO 80306 \\ ${ }^{g}$ Kapteyn Astronomical Institute, P.O. Box 800, 9700 AV Groningen, Netherlands \\ hJet Propulsion Laboratory, MS 264-767, 4800 Oak Grove Drive, Pasadena, CA 91109
}

\begin{abstract}
The Astrobiology Explorer (ABE) is a MIDEX mission concept, currently under Concept Phase A study at NASA's Ames Research Center in collaboration with Ball Aerospace \& Technologies, Corp., and managed by NASA's Jet Propulsion Laboratory. ABE will conduct infrared spectroscopic observations to address important problems in astrobiology, astrochemistry, and astrophysics. The core observational program would make fundamental scientific progress in understanding the distribution, identity, and evolution of ices and organic matter in dense molecular clouds, young forming stellar systems, stellar outflows, the general diffuse ISM, HII regions, Solar System bodies, and external galaxies. The ABE instrument concept includes a $0.6 \mathrm{~m}$ aperture Ritchey-Chretien telescope and three moderate resolution $(\mathrm{R}=2000-3000)$ spectrometers together covering the 2.5-20 micron spectral region. Large format (1024 $\mathrm{x}$ 1024 pixel) IR detector arrays will allow each spectrometer to cover an entire octave of spectral range per exposure without any moving parts. The telescope will be cooled below $50 \mathrm{~K}$ by a cryogenic dewar shielded by a sunshade. The detectors will be cooled to $\sim 7.5 \mathrm{~K}$ by a solid hydrogen cryostat. The optimum orbital configuration for achieving the scientific objectives of the ABE mission is a low background, 1 AU Earth driftaway orbit requiring a Delta II launch vehicle. This configuration provides a low thermal background and allows adequate communications bandwidth and good access to the entire sky over the $\sim 1.5$ year mission lifetime.
\end{abstract}

Keywords: Astrobiology, infrared, Explorers, interstellar organics, telescope, spectrometer, space, infrared detectors

\section{INTRODUCTION}

Space is neither empty nor static, for it is composed of dust, ices, and molecules forged through time by a variety of exotic chemical processes. Over 20 years of combined theory, laboratory simulations, and astronomical observation have shown that organic molecules likely permeate the universe. However, what remains unknown is the abundance, distribution, and identities of these potential building blocks of life. The AstroBiology Explorer (ABE) MIDEX mission concept was developed to allow for the systematic study the nature, evolution, and distribution of organic molecules in the local universe and assess the role that extraterrestrial organics may have played in the development of life.

Previous ground, airborne-, and space-based infrared (IR) instruments have provided glimpses of the rich insights to be gathered from interstellar materials. However, our current understanding of these organics has been limited by observations of materials along only a few lines of sight, and often with limited IR spectral coverage and sensitivity.

Further author information: E-mail Kimberly Ennico (kennico@mail.arc.nasa.gov) 
This field would be greatly enhanced by the collection of a comprehensive spectral database from a set of targets that sample different evolutionary states of a variety of classes of objects. The ABE mission is designed to provide that sample. Additionally, laboratory studies have created a large and growing database of IR spectra of astrophysicallyrelevant organic materials, which, in combination with sophisticated astrochemical theoretical modeling, can be used to interpret ABE data. The ABE MIDEX mission concept was submitted for review in October 2001 and was selected for Concept Phase A study in April 2002. This paper describes the scientific motivation [Section 2] and technical implementation [Section 3] of the AstroBiology Explorer, a modest sized (60 cm diameter), dedicated IR $(2.5-20 \mu \mathrm{m})$ spectroscopic ( $\mathrm{R}=2000-3000)$ observatory to study the evolution of organics in the local universe.

\section{SCIENCE MOTIVATION}

The ABE MIDEX mission concept was designed to answer the key questions:

- How does the composition and nature of prebiotic organic material evolve as it is transported between and through the various environments involved in the lifecycle of interstellar materials?

- How does the abiotic organic material in the Solar System compare with that in the interstellar medium (ISM)?

- How does the prebiotic material in our Galaxy compare with that in other, nearby galaxies?

Establishing the answers to these key questions requires the IR spectra of the organic materials in a wide variety of galactic, extragalactic, and Solar System environments, both to establish their identity and abundance, and to establish the chemical relationships among them. These required measurements from $\sim 2000$ objects are organized into six tasks which are briefly described below. Further details about these tasks are documented in the literature. ${ }^{1}$

\subsection{Evolution of complex organic molecules in the interstellar medium \& in stellar outflows}

Polycyclic aromatic hydrocarbons (PAHs) represent one of the principal forms of complex molecular carbon in space. These molecules are generally thought to be initially produced in the outflows of late type carbons stars forming protoplanetary and planetary nebulae (PPN/PN). ${ }^{2}$ The PAHs are detectable via the characteristic IR emission features they produce as they are excited by UV and visible photons. The population of PAHs will evolve as they are subjected to the increasingly intense radiation field of their central star. ABE will study this evolution by examining the differences in the spectra obtained from PPN and PN that are at different stages of evolution. In addition, the PAH population will be further modified in the ISM by the interstellar radiation field, supernovae shock waves, condensation and irradiation in ices in dense clouds, etc. Thus, ABE will also study PAH evolution by examining PAH emission spectra from HII regions and their boundaries with dense molecular clouds.

\subsection{Distribution of organic compounds in the diffuse interstellar medium}

On the basis of limited observations along a few lines of sight within our galaxy, it is known that $\sim 10 \%$ of carbon in the diffuse ISM is in the form of aliphatic hydrocarbons. ${ }^{3,45}$ These materials appear to be associated with comparable or greater amounts of aromatic hydrocarbons. ABE will use field stars to probe for organic absorption bands along a multitude of lines of sight throughout our galaxy to determine the composition, abundance, and distribution of this material within our galaxy.

\subsection{Determining the evolution of ices and organic matter in dense molecular clouds and star forming regions}

Independent of the formation site and subsequent interstellar evolution of cosmic organics, they must ultimately pass through the dense cloud phase if they are to end up on the surface of a planet. Thus, understanding the environment surrounding forming stellar systems is critical to addressing the role interstellar organics may have played in the origin of life. Dense clouds are especially interesting because laboratory, theoretical, and telescopic observations indicate that complex organic species are created in these environments. ${ }^{6,7}$ ABE will greatly improve our understanding of the molecular nature of these environments by obtaining absorption spectra of embedded solar-mass protostars and background field stars that will probe a large number of lines of sight through several nearby dense clouds in which star formation is occurring. 


\subsection{Organics in the Solar System}

Comets and asteroids, and the dust and meteoroids they produce, probably represent the principal bodies responsible for delivering interstellar materials to early planetary surfaces. Thus, understanding their organic contents is of direct interest to determining the role interstellar materials may have played in the origin of life. ABE will obtain spectra from a number of these objects. ABE will also obtain spectra from the surfaces of icy bodies in the outer solar system (Kuiper Belt Objects, satellites of the gas giants, Pluto and Charon), objects that may either be repositories of interstellar organics or sites on which organics may be formed in situ.

\subsection{Tracing the history of molecular carbon}

ABE will take the spectra of a number of galaxies to examine the their molecular composition as a function of type and cosmic distance. Spectral features such as the PAH emission bands and aliphatic absorption bands have both been seen external galaxies. ${ }^{8}$ Targets include ultraluminous IRAS galaxies, Starburst galaxies, Seyfert galaxies, distant ULGs, and dwarf, S0, spiral, and elliptical galaxies. In addition, ABE will examine a few colliding galaxies and do more detailed spectral mapping of a few nearby galaxies.

\subsection{Tracing deuterium enrichments}

In a number of favorable cases, $\mathrm{ABE}$ will obtain spectra with sufficiently high $\mathrm{S} / \mathrm{N}$ to detect or derive meaningful upperlimits to the $\mathrm{D} / \mathrm{H}$ ratios in the organics. A number of astrochemical processes enrich organics in $\mathrm{D}$ in the ISM and it is the presence of D-enrichments in the organics found in meteorites and interplanetary dust that provides the principal proof that some interstellar organics can survive their incorporation into forming stellar systems and subsequent arrival on the surface of planets. Thus, we hope to better establish the connections between interstellar chemistry and the complex suite of organics found in extraterrestrial materials.

\subsection{Guest Observations}

The core science mission places constraints on the required capabilities of the instrumentation needed to carry out the observational program. An instrument and spacecraft that is ideal for meeting these requirements is described in further detail in Section 3. However, such an instrument would also be capable of making observations of great significance addressing a variety of astrophysical issues not falling directly into the scientific goals and observing programs described above. Therefore, we intend to set aside $\geq 20 \%$ of ABE's total observing time for use by the general astronomical community on a competitive basis. Thus, the capabilities of $\mathrm{ABE}$ will be used to address a wide variety of astrophysical issues that extend well beyond the central scientific goals of the ABE misison.

\section{TECHNICAL IMPLEMENTATION}

\subsection{Scientific Requirements}

The vibrational frequencies associated with the most common chemical bonds between the most abundant elements fall in the middle infrared 2.5 to $20 \mu \mathrm{m}\left(400-500 \mathrm{~cm}^{-1}\right)$ portion of the electromagnetic spectrum [Figure 1]. This is a natural consequence of the masses and interatomic bond strengths of $\mathrm{C}, \mathrm{H}, \mathrm{O}$, and $\mathrm{N}$. While many chemical functional groups and some classes of molecule display distinctive characteristic infrared bands, it is necessary to detect multiple bands of a molecule to derive meaningful information. Thus, the ABE instrumentation must be capable of providing spectral coverage across most or all of this range.

A spectral resolution $(\mathrm{R} \equiv \lambda / \Delta \lambda)$ of about $2000-3000$ is also desirable for this work; it is high enough to resolve almost all the bands produced by organics in solids and provide sufficient detail of gas phase rotational lines and envelopes that they can be separated from the solid state features [Figure 2]. 


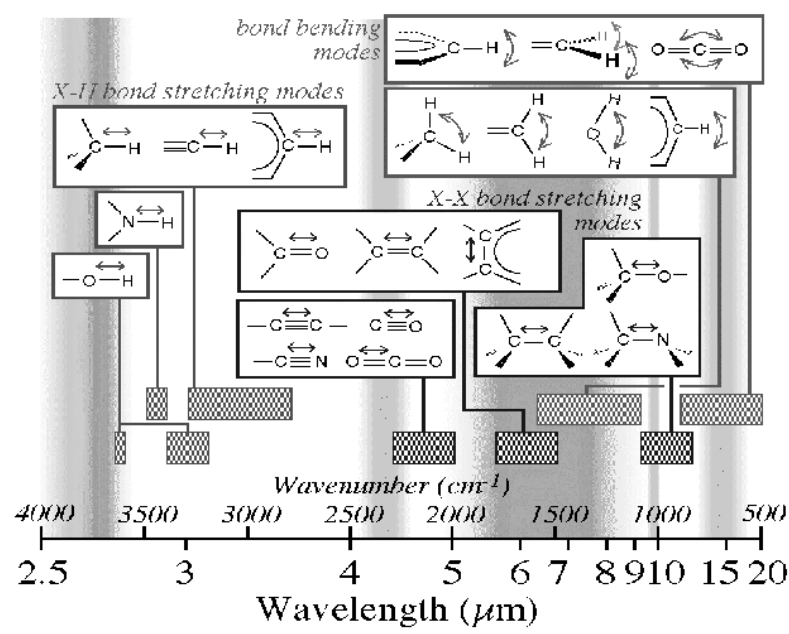

Figure 1. Fundamental vibration frequencies associated with the most common chemical bonds between the most abundant elements populate the $2.5-20 \mu \mathrm{m}$ region. The shaded bands correspond to the wavelength regions that inaccessible from ground-based and/or airborne observatories due to atmospheric $\mathrm{H}_{2} \mathrm{O}$ and $\mathrm{CO}_{2}$.

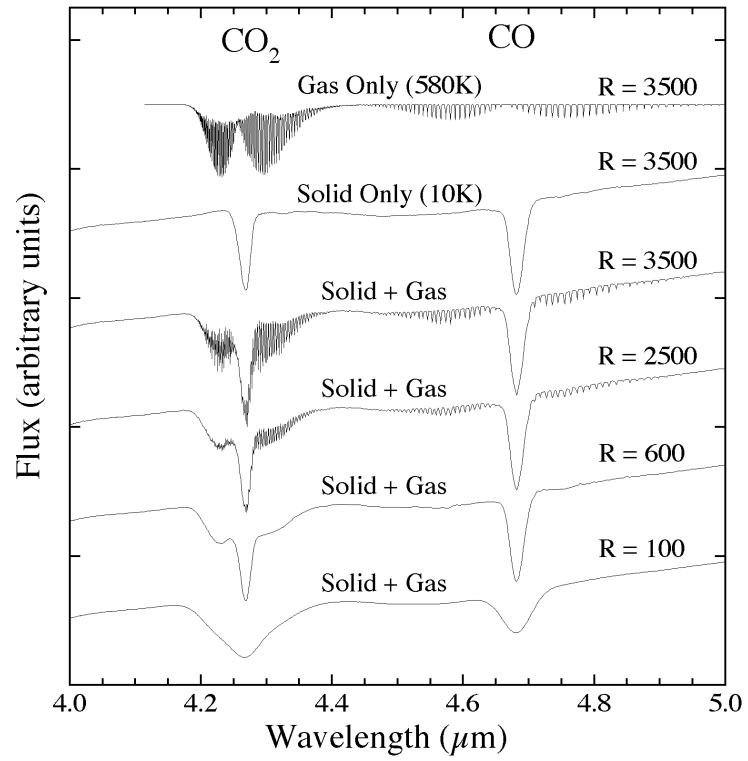

Figure 2. Resolution $R=2000-3000$ is required for detection of organics in solids and provide sufficient detail of gas phase rotational lines.

Additionally, many of the absorption and emission features that will be studied have strengths that are only a few percent of the continuum flux. Moreover, the target list for this mission will contain 2000 objects, many of which are relatively faint, on the order of 0.01-0.10 Jy. Thus, achieving the goals in the ABE science mission will require high sensitivities, with signal-to-noise $(\mathrm{S} / \mathrm{N})$ values on the order of 100 in many cases.

Based on realistic expectations, we anticipate that obtaining the spectra of our target objects with the needed quality will require a mission duration of approximately 1.5 years, a timescale that will allow us to study objects in all parts of the sky.

The main scientific requirements that $\mathrm{ABE}$ will have to meet if it is to properly carry out its science mission are summarized in Table 1.

Table 1. Nominal Scientific Requirements for the AstroBiology Explorer

\begin{tabular}{|l|l|}
\hline Wavelength Coverage & $2.5-20 \mu \mathrm{m}$ (no gaps) \\
\hline Spatial Resolution (slit width) & 8.3 "' \\
\hline Spectral Resolution $\mathrm{R} \equiv \lambda / \Delta \lambda$ & $\begin{array}{l}2500-3500(2.5-4.8 \mu \mathrm{m}) \\
2000-3000(4.8-20 \mu \mathrm{m})\end{array}$ \\
\hline Slit length & $>33$ " \\
\hline $\begin{array}{l}\text { Sensitivity @ } \lambda=10 \mu \mathrm{m} \\
\text { in } 1 \mathrm{hr}(\mathrm{S} / \mathrm{N}=100)\end{array}$ & $50 \mathrm{mJy}$ \\
\hline Calibration & $\begin{array}{l}\text { Absolute flux accuracy } 25 \% ; \\
\text { Wavelength dependent relative } \\
\text { accuracy } 10 \%\end{array}$ \\
\hline Operational Lifetime & $\sim 1.5$ yrs \\
\hline Pointing Stability & $<3$ " for a 1000 sec exposure \\
\hline Spatial coverage of slits on sky & Co-spatial on sky \\
\hline
\end{tabular}

\subsection{Optical Design}

The ABE resolution requirement is met by an effective aperture of approximately $0.6 \mathrm{~m}$. The ABE optical design employs a f/6.7, $60 \mathrm{~cm}$ diameter diffraction-limited Ritchey-Chretien telescope with a 4.0 m effective focal length. 
[Figure 3]. The telescope resides behind a sunshade and is further cooled below $50 \mathrm{~K}$ by solid hydrogen [Section 3.4]. The telescope mirrors will be $6061 \mathrm{Al}$ diamond turned and post-polished.

The 2.5-20 $\mu \mathrm{m}$ continuous desired wavelength range spans three full octaves. To avoid overlapping of orders, the ABE optical design uses three separate spectrometers. These three "arms" share the same telescope field of view. Two dichroic beamsplitters are used after a single slit to separate the light into the three bands. The slit size is chosen to maximize sensitivity across the large spectral range. Our baseline concept consists of an 8.3" wide slit, which allows the detection of the full point spread function of the Airy disk at $10 \mu \mathrm{m}$ for a $60 \mathrm{~cm}$ telescope. We have found that larger slits, although lowering slit loss effects at the long wavelength end, will decrease our estimated ABE sensitivity significantly at the longer wavelengths. The slit is mapped to a two-to-three detector pixel width in order to maintain full spatial resolution and high optical throughput without a noise penalty when background limited.

Since we require at least $2 \mathrm{R}$ pixels in the spectral direction in order to Nyquist sample the spectrum where $\mathrm{R} \equiv \lambda / \Delta \lambda=$ 2000-3000, we need large format IR detector arrays. Both near and mid-IR detector arrays up to $1024 \times 1024$ pixels in size are expected to be available well before launch in 2007 or 2008 [Figure 4]. The ABE optical design utilizes a single $1024 \times 1024$ pixel device per spectrometer arm. The $\mathrm{R}=2000-3000$ spectra are cross-dispersed onto the arrays, providing a more compact and lower mass design compared with one having multiple detector arrays arranged linearly in the dispersion direction [Figure 5].

All three spectrometers share the same fundamental design. Each shares the entrance slit located at the focal plane and a common collimator. Within each arm, light next passes through dichroic beamsplitter(s) and is dispersed by an "echellette" and cross-disperser gratings and finally focused onto a detector by the camera optics. The common collimator is a single element parabola, while the cameras consist of a few reflective surfaces and a single refractive element ( $\mathrm{ZnSe}$ lens for the 2.5-5 $\mu \mathrm{m}$ and 5-10 $\mu \mathrm{m}$ channels, KRS-5 for the 10-20 $\mu \mathrm{m}$ channel). The design is diffraction limited at all wavelengths.

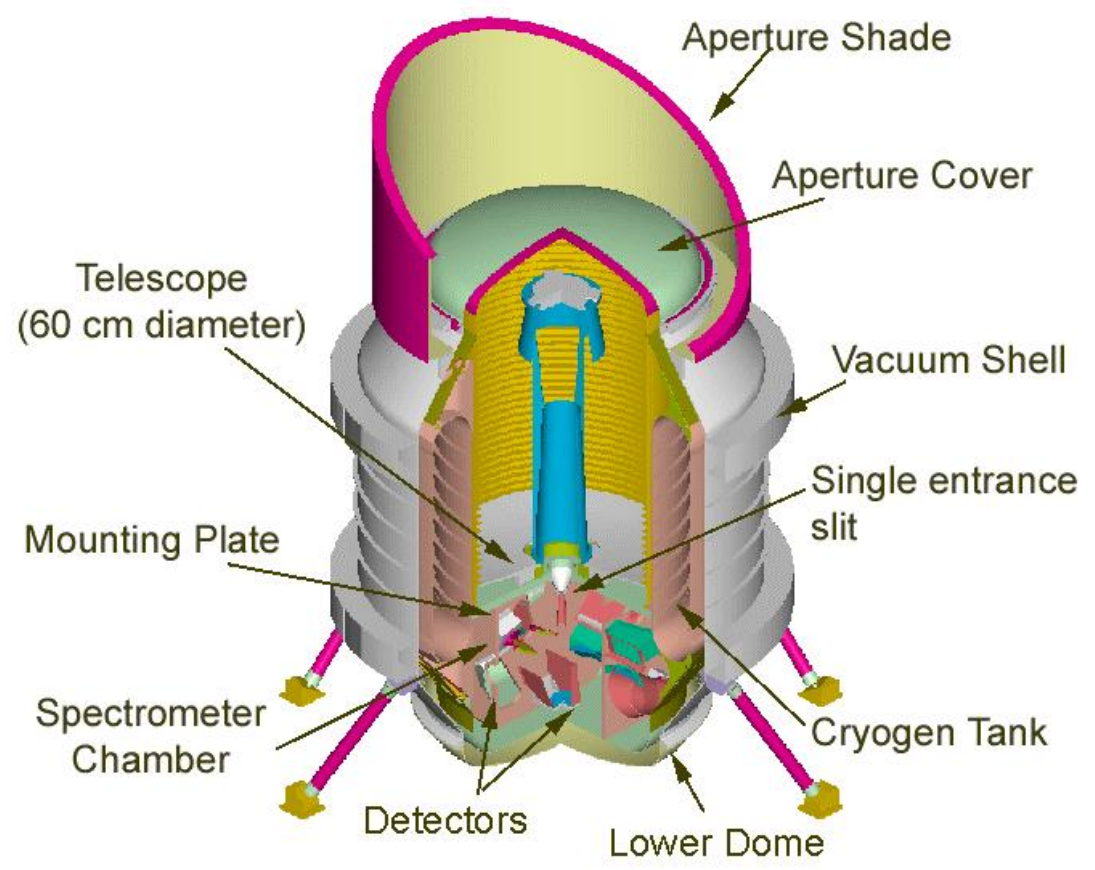

Figure 3. The ABE instrument concept. 


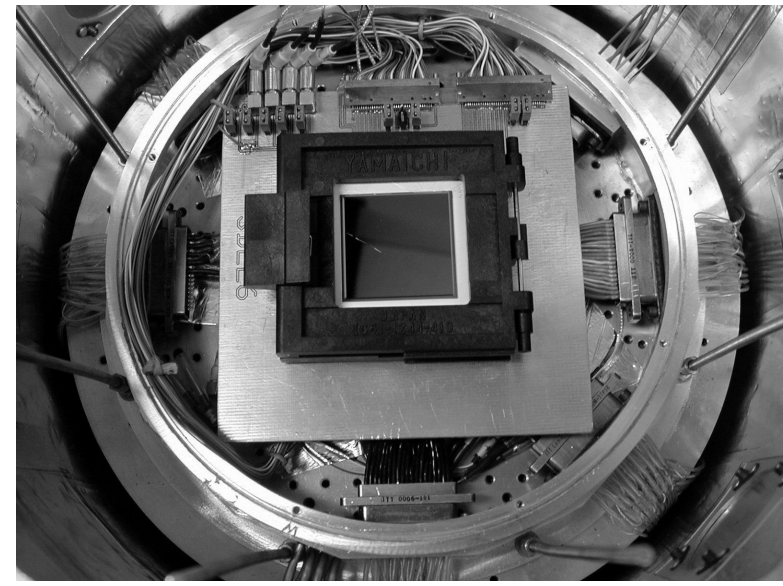

Figure 4. $1024 \times 1024$ pixel Si:As hybrid under test at the NASA Ames IR detector lab. These large format devices are integral to the ABE design.

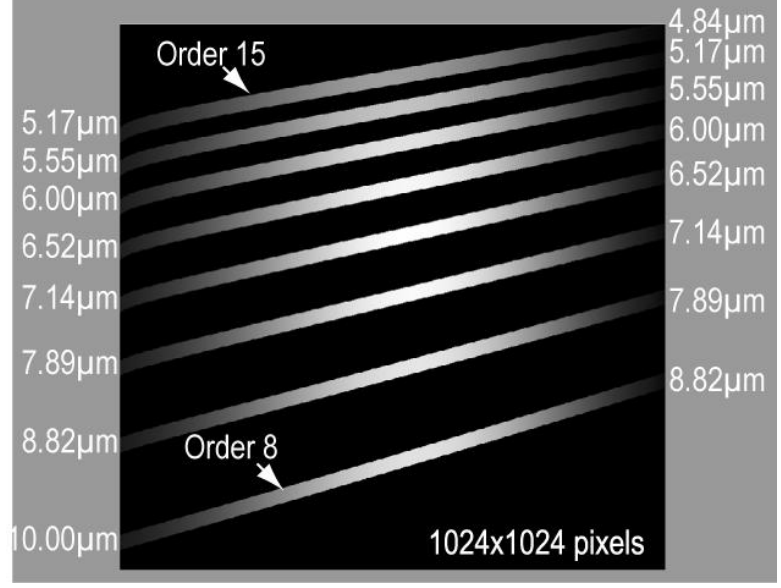

Figure 5. Cross-dispersed echelle layout of the 5-10 $\mu \mathrm{m} \mathrm{ABE}$ spectra on a single $1024 \times 1024$ format FPA.

\subsection{Infrared Detectors}

In addition to meeting the above format $(\geq 1024 \times 1024$ pixels) and wavelength $(2.5-20 \mu \mathrm{m})$ requirements, the $\mathrm{ABE}$ detectors need to have high quantum efficiency $(\eta>50 \%)$ and low noise in order to meet the ABE sensitivity requirements [see Figure 8].

Ideally, total detector noise [defined as (readnoise ${ }^{2}+$ dark_counts) $^{0.5}$ ] should be less than the photon flux background on the detectors at all wavelengths. With a cold telescope $(\mathrm{T}<50 \mathrm{~K})$, ABE's expected performance will be dominated by detector properties, except for bright sources where the shot noise from the object itself will dominate. At a resolution of $\mathrm{R}=2500$, the expected photon background (for the ecliptic pole, a $\mathrm{T}=50 \mathrm{~K}, 60 \mathrm{~cm}$ diameter telescope, and minimal scattered light) per pixel detected with a conservative optical system (DQE $\sim 0.7$, optics efficiency $\sim 0.3$ ), can be as low as $0.07,0.2,4$ and 26 photons per second at wavelengths of $2.5,5,10$, and $16 \mu \mathrm{m}$, respectively, when mapped to a $2 \times 2$ resolution element for a 8 " wide slit. Thus, attempts to minimize both detector dark currents (electrons per second per pixel) and read out noise will be required to optimize ABE's performance.

The preferred detector array technologies for ABE are Raytheon Infrared Operations' (RIO) 1024 x 1024 pixel format $\mathrm{InSb}$ and $\mathrm{Si}$ :As detector arrays, for the $2.5 \mu \mathrm{m}$ and 5-20 $\mu \mathrm{m}$ spectral ranges, respectively. Both technologies have demonstrated excellent performance in the Space Infrared Telescope Facility (SIRTF) instruments in 256 x 256 pixel formats and have successfully passed a number of rigorous characterization and radiation testing. ${ }^{9,10}$ The larger $1 \mathrm{M}$ pixel format devices are currently under development for the Next Generation Space Telescope (NGST) and Origins observatories (i.e., low background, low noise, high sensitivity) under the direction of NASA Ames Research Center.

We at Ames have recently tested the first generation 1024 x $1024 \mathrm{Si}$ :As hybrid device, after a pre-selection of candidate cryo-processed readout designs. Dark currents $<0.1 \mathrm{e} / \mathrm{s}$ have been measured at $7 \mathrm{~K}$. Power dissipation, for a full-frame scan time of $3.8 \mathrm{~s}$, was measured to be below $500 \mu \mathrm{W} .{ }^{11}$ These two items are lower than required for ABE [Table 2]. The read noise measured, $\sim 35$ electrons rms (Fowler 4), although adequate for ABE's required sensitivity, is too high for NGST. Raytheon is presently developing second generation multiplexer designs for decreased noise performance. Such devices are presently under test. These Si:As detector arrays have good quantum efficiency ( $60-70 \%$ non AR coated, $>80 \%$ with appropriate AR coatings) over the 5-25 $\mu \mathrm{m}$ range. Unfortunately, the quantum efficiency degrades rapidly for wavelengths shorter than $4 \mu \mathrm{m}$. For ABE, where sky backgrounds at wavelengths $\lambda<5 \mu \mathrm{m}$ are negligible, observations will likely be detector-noise limited there. The Si:As detector was found to be inadequate in performance to meet ABE's sensitivity requirements over $2.5-5 \mu \mathrm{m}$.

ABE's shortest $(2.5-5 \mu \mathrm{m})$ wavelength spectrometer utilizes a single $1024 \times 1024$ pixel InSb device, with the same multiplexer as for the Si:As device above, also supplied by RIO. It has good quantum efficiency ( $\sim 5 \%$ with appropriate AR coating) in this region, read-noise $\sim 8$ electrons rms (Fowler 32), and dark currents $<0.05$ electrons/second. ${ }^{12}$ These devices operate at $\mathrm{T} \leq 32-35 \mathrm{~K}$ to maintain this low dark current. 
Table 2. ABE Detector Performance Requirements

\begin{tabular}{|l|l|l|}
\hline & \multicolumn{1}{|c|}{$\begin{array}{c}2.5-5 \mu \mathrm{m} \\
\text { spectrometer }\end{array}$} & \multicolumn{1}{|c|}{$\begin{array}{c}\text {-10 } \mu \mathrm{m} \text { and } 10-20 \mu \mathrm{m} \\
\text { spectrometers }\end{array}$} \\
\hline Type & InSb & Si:As \\
\hline Format & $\geq 1024 \times 1024$ & $\geq 1024 \times 1024$ \\
\hline Unit cell size & $27 \mu \mathrm{m}$ & $27 \mu \mathrm{m}$ \\
\hline Wavelength range & $2.5-5 \mu \mathrm{m}$ & $5-20 \mu \mathrm{m}$ \\
\hline Operating temperature & $\mathrm{T} \leq 35 \mathrm{~K}$ & $\mathrm{~T} \leq 8 \mathrm{~K}$ \\
\hline QE & $>80 \%$ & $>60 \%$ \\
\hline Read Noise & $\leq 5 \mathrm{electrons}$ & $\leq 35 \mathrm{electrons}$ \\
\hline $\begin{array}{l}\text { Dark Current at } \\
\text { operating temperature }\end{array}$ & $\leq 0.1 \mathrm{e} / \mathrm{s}$ & $\leq 1 \mathrm{e} / \mathrm{s}$ \\
\hline Power dissipation & $<2 \mathrm{~mW}$ & $<2 \mathrm{~mW}$ \\
\hline Well capacity & $>2.5 \mathrm{e} 5$ electrons & $>2.5 \mathrm{e} 5$ electrons \\
\hline
\end{tabular}

Although the ABE instrument concept includes two different detector types, both are manufactured by the same company (Raytheon) and share the same read-out multiplexer. This allows for common electronics to be used for all ABE focal planes, yielding development simplification and cost savings.

The main detector properties required by $\mathrm{ABE}$ to achieve its science mission are summarized in Table 2.

\subsection{Thermal Design}

Calculations show that the ABE sensitivity requirements can be met with

a cryogenically cooled instrument design. The telescope and spectrometer optics and IR array detectors are all contained within a cryogenic dewar [Figure 3]. The outer shell of the dewar is protected from solar radiation by a sunshade, and an aperture shade minimizes radiative loading of the dewar when tipped toward the Sun. The dewar is thermally isolated from the spacecraft bus by minimizing connections and the careful placement of insulation. The anti-sun side of the dewar is coated to act as a radiator, cooling the dewar outer shell.

Table 3. The ABE Thermal Design

\begin{tabular}{|l|l|}
\hline Cryogen type & Solid Hydrogen \\
\hline SH2 tank volume & 267 liters \\
\hline $\begin{array}{l}\text { SH2 tank operating } \\
\text { temperature }\end{array}$ & $7.2 \mathrm{~K}$ \\
\hline Cryogen lifetime & $>1.5$ years \\
\hline Cryostat dimensions & $\begin{array}{l}\sim 110 \mathrm{~cm} \\
\text { diameter, } \\
>49 \mathrm{~cm} \text { length }\end{array}$ \\
\hline Instrument cavity dimensions & $\begin{array}{l}60 \mathrm{~cm} \text { diameter, } \\
43 \mathrm{~cm} \text { length }\end{array}$ \\
\hline Vapor cooled shields & None \\
\hline Vacuum shell temperature & $\leq 65 \mathrm{~K}$ \\
\hline Launch Pad hold time & $>12$ hours \\
\hline $\begin{array}{l}\text { Telescope, instrument, } \\
\text { cryostat, aperture shade mass }\end{array}$ & $\begin{array}{l}375 \mathrm{~kg} \text { (incl. 30\% } \\
\text { margin) }\end{array}$ \\
\hline
\end{tabular}

To meet our sensitivity requirements, the ABE sunshade must be cooled to $<70 \mathrm{~K}$, and the telescope primary and secondary mirrors must be cooled to $<50 \mathrm{~K}$. The Si:As detector arrays are cooled to $7.5 \mathrm{~K}$, and the InSb device is actively heated to $15 \mathrm{~K}$. In addition to meeting the above primary, sunshade, and detector thermal requirements, high emissivity elements in the spectrometers must be cooled to $\mathrm{T} \leq 18 \mathrm{~K}$ to limit self-generated thermal backgrounds that would degrade the sensitivity.

After performing several trade studies, the most cost-effective cryogen choice for $\mathrm{ABE}$ is solid hydrogen. The $\mathrm{ABE}$ cryostat design utilizes a single-stage solid hydrogen dewar, oversized for a large lifetime margin, no vapor cooled shields, all aluminum construction to match the telescope materials, and a torodial tank design with removable vacuum shell domes to mitigate complexity during integration and test of the ABE payload. Top level details of the ABE cryostat design are described in Table 3.

Solid cryogen dewars have considerable flight history and experience base and are the best option for an Explorer class mission that can afford very little development cost or schedule. Mechanical closed cycle coolers have the potential for longer lifetimes than stored cryogen coolers, but they induce vibration and there are no flight-qualified models that meet the ABE thermal requirements. Thus, they are currently not realistic candidates for a very low temperature Explorer-class mission.

\subsection{Spacecraft Design}

The top-level spacecraft and mission requirements for the ABE mission are provided in Table 4. The spacecraft bus will contain all of the uncooled spacecraft components for command and data handling/storage, power, pointing, and control and communication. This portion of the spacecraft will be isolated from the passively cooled telescope and instrument deck by a standoff support structure and thermal insulation [Figure 6].

The thermal control system uses passive thermal design techniques (sun shades, multilayer insulation blankets, paint and thermal tape, and localized radiators), coupled with limited-use active heaters, to allow continuous operations in all 
allowed sun angles and modes of operation. A sunshade will be used to provide passive cooling of the cryostat outer shell to $<70 \mathrm{~K}$. The sunshade, backed by insulation, extends above the top deck of the spacecraft and reduces radiative heat transfer to the cryostat. As a result, the spacecraft attitude during flight must be within $\sim \pm 30^{\circ}$ from normal to the spacecraft-sun line. Thus, at any given time, the spacecraft will only be able to observe objects within an annulus on the sky, which, during the course of the mission, will sweep across the entire sky.

Power is generated by the solar array using high efficiency triple-junction solar cells. To enable the solar cells to operate at their peak efficiency, the solar array is deployed adjacent to and below the spacecraft, allowing the back of the array to radiate to space. A small Nickel Hydrogen $(\mathrm{NiH})$ battery will be used for power during initial orbit insertion and for safe hold mode. Battery power will not be required during normal operations since the spacecraft will be in an Earth driftaway orbit and no solar eclipses will occur during the mission.

The spacecraft will be 3-axis stabilized using reaction wheels. The instrument pointing stability requirements of 1 arcsec will be met using an inertial reference unit and a bore-sighted star tracker. Peak-up methods using the spectral science data will be used for fine tuning pointing, if needed. Sun sensors are also used to keep the solar array pointed at the sun. Three reaction wheels will be used for redundancy.

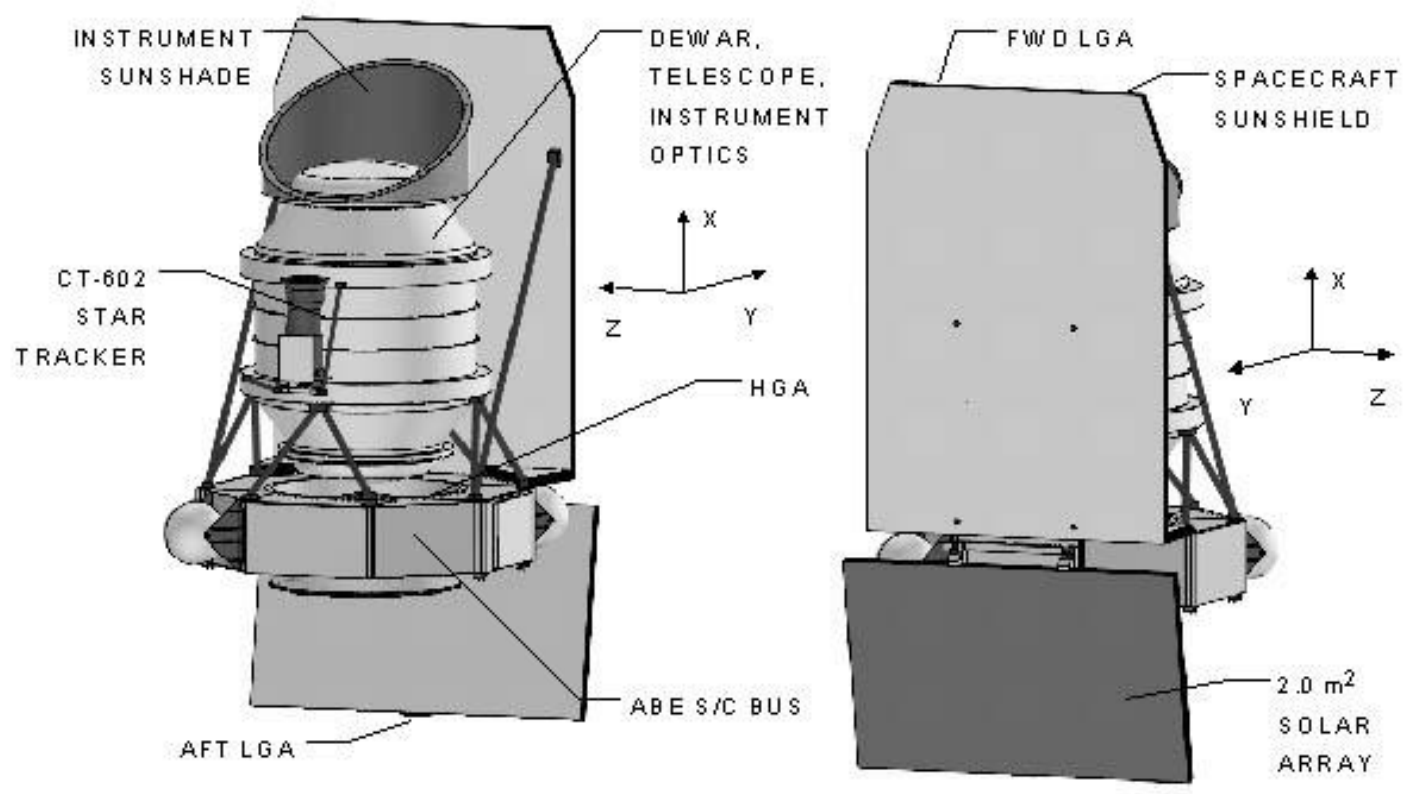

Figure 6. The ABE spacecraft concept.

A cold-gas propulsion system will be employed to offload momentum build-up due to external torques, such as initial upperstage tip-off separation and solar pressure. The cryogenic $\mathrm{H}_{2}$ boiloff from the optics dewars will be 'equal reaction' vented so that it imparts no net contribution to the spacecraft momentum.

Communication with $\mathrm{ABE}$ would be carried out using an X-band transponder with a $25 \mathrm{~W}$ amplifier and a high-gain antenna mounted on the base of the spacecraft bus. The high-gain antenna will be mounted to the bus with a 2-axis gimbal to enable data downlink and command uplink contemporaneous with ABE data-taking observations. At the end of the ABE mission the spacecraft will be about $3 \times 10^{7} \mathrm{~km}$ from the Earth. At this distance, the DSN standard deep space transponder can be run at its maximum downlink capability of $4.4 \mathrm{Mbps}$ for the entire mission when tracked from the $34 \mathrm{M}$ HEF antennae, well over the predicted ABE rates (1Mbps). A low gain omni-directional X-band antenna receiver would also be included to listen for unscheduled ground commands.

The ABE command and data handling $(\mathrm{C} \& D H)$ subsystem uses a bus-based architecture to provide a significant level of software-based, higher order processing within a modular, fully integrated spacecraft control unit (SCU) using a RAD 
750 processor. The SCU localizes all processing needs in a central computer with remote sensors and actuators. This concept differs from previous avionics architectures by allowing higher order "processing" functionality previously accomplished in hardware to be transferred to software applications on the central computer. This transfer allows a reduction in the number of recurring hardware components and provides functional flexibility through data table configurable software applications.

Table 4. ABE Spacecraft System Requirements

\begin{tabular}{|l|l|}
\hline Launch vehicle & Delta II 2425 with 2.9m fairing \\
\hline Orbit & $\begin{array}{l}\text { Heliocentric Drift-Away } \\
\text { Max earth range 0.21 AU at 18 months }\end{array}$ \\
\hline Mission Lifetime & $>18$ months \\
\hline Attitude Control & 3 -axis stabilized \\
\hline Pointing Accuracy & $3 \operatorname{arcsec}(3 \sigma)$ \\
\hline Pointing Stability & $1 \operatorname{arcsec} / 1000 \mathrm{~s}(3 \sigma)$ \\
\hline Pointing Jitter & 1 arcsec ptp \\
\hline Telecommunications & X-band into DSN 34m \\
\hline Payload Data Volume & 6 Gbit / day \\
\hline Observatory Power & $300 \mathrm{~W}$ (observe mode) \\
\hline Observatory Mass & $608 \mathrm{~kg}$ (incl. 25\% contingency) \\
\hline
\end{tabular}

\subsection{Mission Design \& Operations}

The current mission plan has the ABE spacecraft launched from the Eastern Test Range (Cape Canaveral) by a Delta-II 2425 three stage expendable launch vehicle. An Earth-trailing trajectory has been selected to simplify flight operations while satisfying all mission design requirements [Table 5]. This trajectory minimizes pointing restrictions, avoids the Earth's thermal and radiation environments, and does not require orbital maintenance. An Earth-trailing trajectory meets all spacecraft pointing requirements for power, thermal, communication, and telescope orientation. The launch trajectory is characterized by a C 3 of $0.4 \mathrm{~km}^{2} / \mathrm{sec}^{2}$, slightly over Earth escape, to account for the three-body gravitational affects. Within $\sim 30$ days after launch it enters a heliocentric orbit in the ecliptic plane with a period of $\sim 366$ days. In such an orbit, similar to that of SIRTF, the spacecraft only slowly drifts away from the Earth, never getting more than

Table 5. ABE Mission/ Orbit Requirements Summary.

\begin{tabular}{|l|l|}
\hline $\begin{array}{l}\text { Launch date } \\
\text { (nominal) }\end{array}$ & $\begin{array}{l}\text { 15 June 2007 (maximizes galactic } \\
\text { plane view) }\end{array}$ \\
\hline Launch vehicle & Delta II 2425 with 2.9m fairing \\
\hline Launch site & Cape Kennedy \\
\hline Launch window & 30 sec, once per day \\
\hline Orbit type & $\begin{array}{l}\text { Circular, heliocentric (drift-away), } \\
\text { Earth trailing }\end{array}$ \\
\hline Semi-major axis/ecc. & $1.001 \mathrm{AU} / 0.037$ \\
\hline Orbit period & 365.9 days \\
\hline Min/max Sun range & $0.9645 \mathrm{AU} / 1.0379 \mathrm{AU}$ \\
\hline Max Earth range & $0.21 \mathrm{AU}$ \\
\hline
\end{tabular}

0.12 AU from the Earth in its first year and never more than 0.22 AU over two years.

$\mathrm{ABE}$ can, in principle, be launched at anytime of the year. However, given that the mission has a nominal lifetime of about 1.5 years and that the list of target objects is significantly biased towards objects within our own galaxy, an optimal observation strategy would use a launch time when the annulus first viewable by the spacecraft is just beginning to sweep across the inner galaxy. This would allow the most target intensive portions of the sky to be observable at least twice, once early in the mission and once late in the mission. Within this broad requirement, the launch time can be selected to minimize the impact of launch dispersions as there will be no propulsion on the spacecraft for orbital corrections. A nominal launch date of June 15, 2007, has been chosen to maximize galactic plane view.

The ABE Mission Operations Center (MOC) would provide for standard flight operation facilities and activities necessary to maintain and control the spacecraft, support science and engineering data acquisition, and provide for data processing and distribution of data to the users. Spacecraft maintenance includes tasks for pointing and orientation control, communications for command and telemetry, orbit determination for tracking and the onboard spacecraft ephemeris, and momentum off-loading. Data acquisition will consist of both telemetry to monitor the status of the 
spacecraft subsystems and science data acquisition. Typical ABE flight operations will include the preparation, transmission, and verification of command memory loads used by the spacecraft to control its subsystems and instrument observations. The ABE Science Operations Center (SOC), in addition to target observation planning, will analyze, archive and distribute science data together with ancillary data such as spacecraft orientation and flight path information. Mission operations for the ABE project would use the facilities and personnel of the DSN for the tracking and data acquisition (TDA) support. Both telemetry and command capability will be required. Figure 7 summarizes the main components of the ABE MOC and SOC.

Flight operations would be controlled from a spacecraft operations center that houses the computers, communications, and software needed to support real-time monitoring and control. Operation plans must specify operations procedures, command sequences, and payload performance requirements to evaluate real-time spacecraft and instrument status. The spacecraft operations center will also contain a science data unit responsible for the project science database and the ancillary trajectory and spacecraft pointing information. The science data unit would also be responsible for science data processing, science data archiving, and science data distribution.

The nominal ABE mission operations system would consist of a small organization reporting directly to the project manager and the mission director. Two primary teams, Mission Operations and Science, will be responsible for realtime spacecraft operations. A support organization, the ground data system, would coordinate the DSN tracking and the data flow requirements.

\subsection{Comparison with other missions}

As an IR spectroscopic mission, ABE is most closely related to the Infrared Space Observatory (ISO), the Space Infrared Telescope Facility (SIRTF), and the Stratospheric Observatory for Infrared Astronomy (SOFIA). ABE will provide higher resolution than ISO's Short Wavelength Spectrometer (SWS) (ABE R>2500; SWS typically R=500, rarely

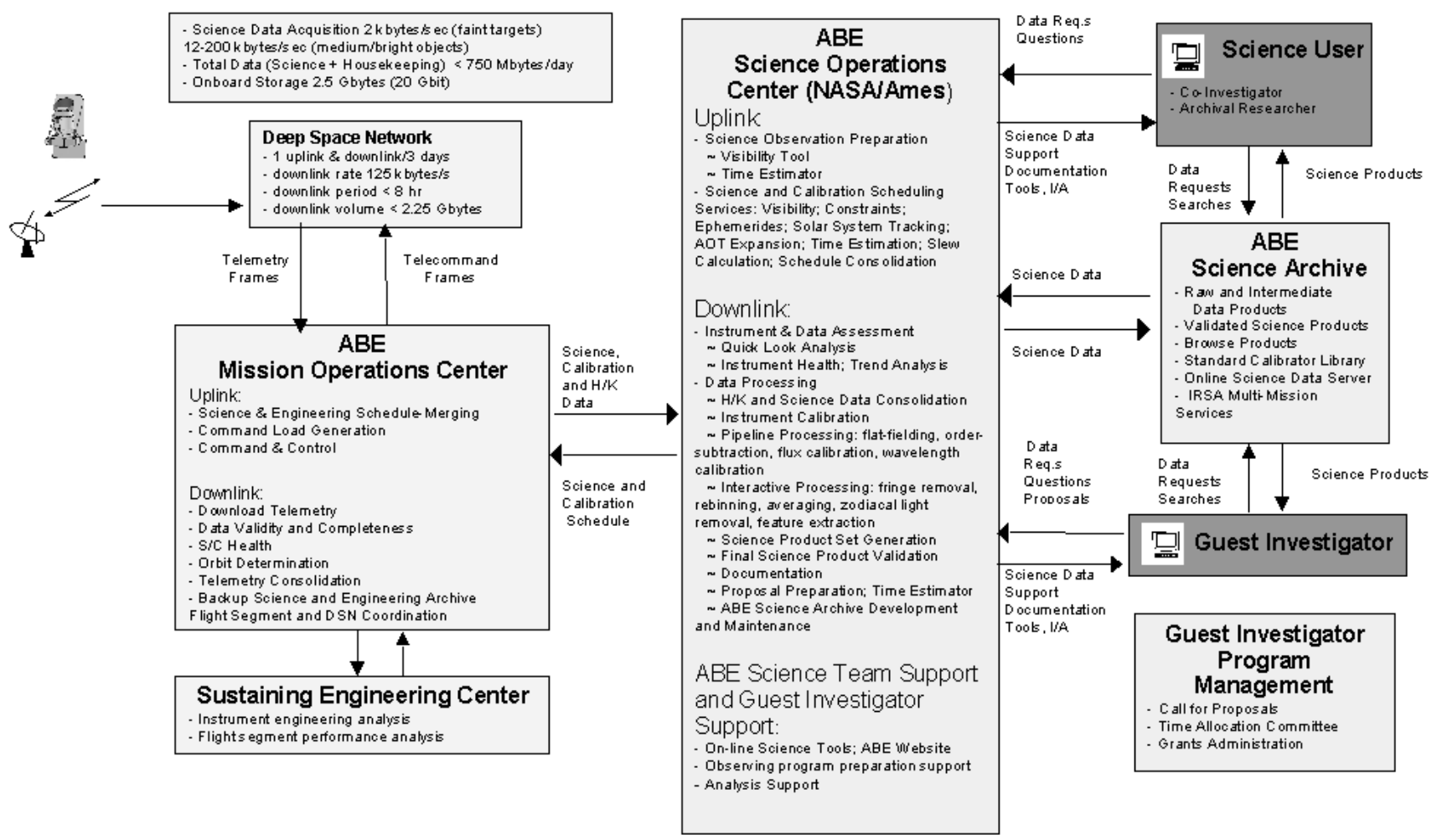

Figure 7. The data flow for the ABE mission operations, science observations, analysis, and archiving. 
$\mathrm{R}=2000$ ), and also be $\sim 100$ times more sensitive than ISO [Figure 8].

The ABE mission should interact synergistically with the SIRTF program. SIRTF will excel at longer IR wavelengths, whereas ABE will excel at shorter IR wavelengths. ABE would fly towards the end of the SIRTF mission and thus be able to follow up in detail on SIRTF discoveries with promising astrobiological implications. ABE, however, will have scientific advantages over SIRTF for molecular Astrobiology from its significantly higher resolving power (ABE

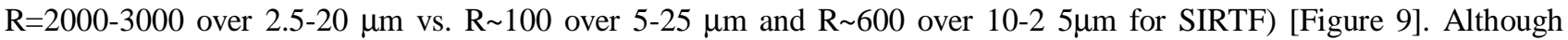
SIRTF has a larger aperture than ABE, advances in detector technology lead us to estimate that the two systems will have equivalent sensitivities in the wavelength overlap region.

SOFIA will also be operational during the ABE mission and, like SIRTF, its scientific program is expected to interact synergistically with ABE's. SOFIA will have access to much, but not all, of the spectral range observed by ABE. SOFIA will not be able to observe in the $\mathrm{CO}_{2}$ bands as $\mathrm{ABE}$ can. We've modeled the performance of an ABE-comparable, midIR spectrometer optimized for SOFIA and, under ideal conditions, we predict that such an instrument would be over 100x less sensitive than ABE [Figure 9]. In addition, since SOFIA operates within the Earth's atmosphere, telluric obscuration [see Figure 1] will prevent SOFIA from addressing some of ABE's science tasks.

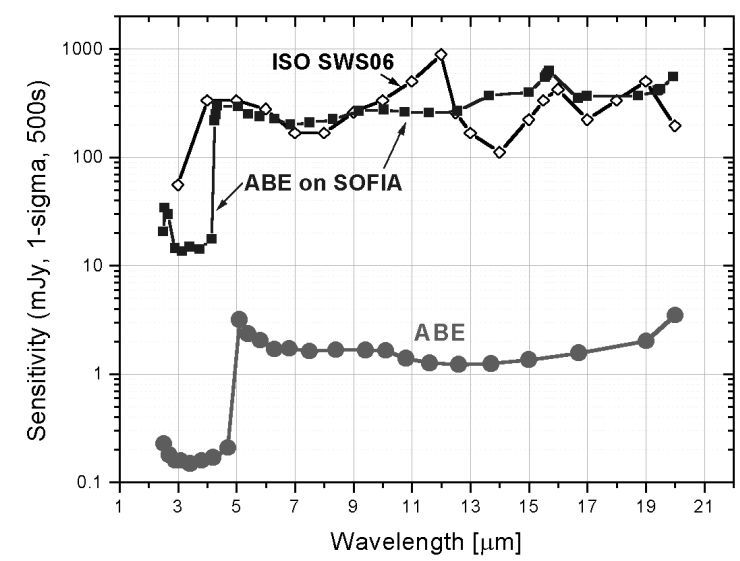

Figure 8. The modeled wavelength-dependent sensitivity of the AstroBiology Explorer compared with other IR spectroscopic platforms of similar spectral resolution. 'ABE on SOFIA' refers to an ABE-comparable, mid-IR spectrometer optimized for SOFIA. More sensitive observatories have lower mJy sensitivities.

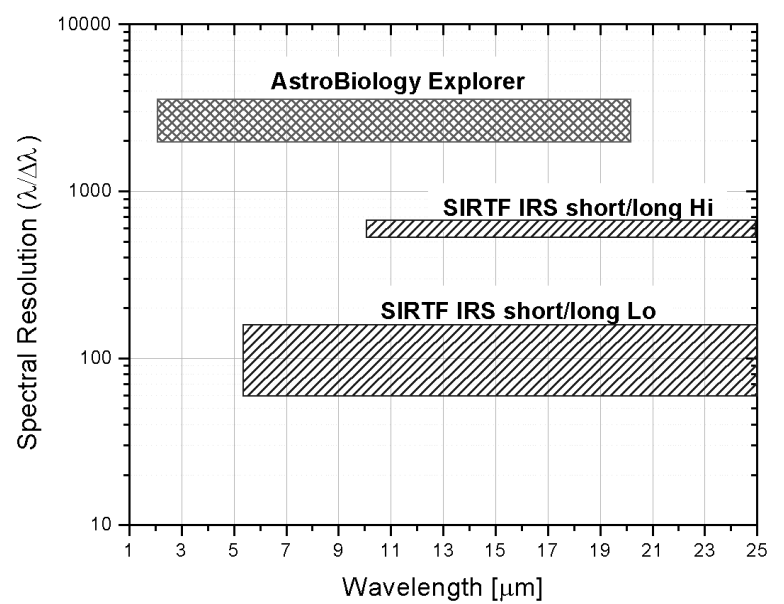

Figure 9. Comparison of the ABE mission concept with existing and planed space and airborne infrared platforms.

\section{TEAM MEMBERS}

The ABE team is shared among three players, NASA Ames Research Center (lead), Ball Aerospace \& Technologies, Corp., and NASA's Jet Propulsion Laboratory (JPL). Ball Aerospace is the prime contracting partner for the ABE mission, and will lead all design, development, building, testing and delivery of the ABE payload. JPL is providing oversight management to the ABE mission. NASA Ames Research Center, under the direction of the PI Scott Sandford, will lead the science planning and science operations center, provide for testing and analysis of the focal planes for flight selection, coordinate EPO activities, and provide management of the instrument payload.

\section{SUMMARY}

It is now known that a significant portion of the cosmic inventory of the elements $\mathrm{C}, \mathrm{O}, \mathrm{N}$, and $\mathrm{H}$ in space are incorporated into a variety of volatiles and organics that are of biogenic interest. However, much remains to be learned about the inter-relationships of these materials and about how they are formed and evolve in space. In this paper have briefly described a potential new MIDEX-class space mission, the AstroBiology Explorer (ABE), consisting of a relatively modest dedicated space observatory having a $60 \mathrm{~cm}$ aperture and spectrometers capable of covering the 2.5-20 $\mu \mathrm{m}$ spectral range at a resolution of $\mathrm{R}=2000-3000$. Such a system would be capable of addressing outstanding 
problems in Astrochemistry and Astrophysics that are particularly relevant to Astrobiology and addressable via astronomical observation. The observational program of this mission would make fundamental scientific progress in each of the key areas of the cosmic history of molecular carbon, the distribution and chemistry of organic compounds in the diffuse and dense interstellar media, the evolution of ices and organic matter in young planetary systems, and the deuterium enrichments in ices, PAHs, and diffuse medium organic refractory materials. ABE could make fundamental progress in all of these areas by conducting an approximately one year mission to obtain a coordinated set of infrared spectroscopic observations of approximately 2000 galaxies, stars, planetary nebulae, and young stellar systems. The ABE MIDEX mission was selected for Concept Phase A study in April 2002.

\section{REFERENCES}

${ }^{1}$ Sandford, S., Allamandola, L., Bregman, J., Ennico, K., Greene, T., Hudgins, D., Strecker, D., "Identifying Organic Molecules in Space - The AstroBiology Explorer (ABE) MIDEX Mission Concept." In "Instruments, Methods, and Missions for Astrobiology IV," eds. R. B. Hoover, G. V. Levin, R. R. Paepe, \& A. Y. Rozanov, Proc. SPIE 4495, pp. 170-181, 2002.

2 Allamandola, L.J., Tielens, A.G.G.M., Barker, J.R., "Interstellar Polycyclic Aromatic Hydrocarbons: the Infrared Emission Bands, the Excitation-Emission Mechanism and the Astrophysical Implications," Ap. J. Suppl. Ser. 71, pp. 733-755, 1989.

${ }^{3}$ Sandford, S.A., Allamandola, L.J., Tielens, A.G.G.M., Sellgren, K., Tapia, M., Y. Pendleton, Y., "The Interstellar C-H Stretching Band near $3.4 \mu \mathrm{m}$ : Constraints on the Composition of Organic Material in the Diffuse Interstellar Medium," Astrophys. J. 371, pp. 607-620, 1991.

${ }^{4}$ Pendleton, Y.J., Sandford, S.A., Allamandola, L.J., Tielens, A.G.G.M., Sellgren, K., "Near-infrared absorption spectroscopy of interstellar hydrocarbon grains," Astrophys. J. 437, pp. 683-696, 1994.

${ }^{5}$ Sandford, S.A., Pendleton, Y.J., Allamandola, L.J., "The Galactic Distribution of Aliphatic Hydrocarbons in the Diffuse Interstellar Medium," Astrophys. J. 440, pp. 697-705, 1995.

${ }^{6}$ Whittet, D.C.B., Schutte, W.A., Tielens, A.G.G.M., Boogert, A.C.A., de Graauw, Th., Ehrenfreund, P., Gerakines, P.A., Helmich, F.P., Prusti, T., van Dishoeck, E.F., "An ISO SWS view of interstellar ices - First results," Astron. Astrophys. 315, pp. L357-L360, 1996.

7 Tielens, A.G.G.M., Allamandola, L.J., "Composition, structure, and chemistry of interstellar dust," in Interstellar Processes, D. Hollenbach and H. Thronson, eds., (D. Reidel: Dordrecht), pp. 397-469, 1987.

${ }^{8}$ see special issue on First ISO Results, Astron. Astrophys. 315, 1996.

${ }^{9}$ Pipher, J.L., Forrest, W.J ,. Glaccum, W.J, Benson, R.G., Krebs, D.J.,. Jhabvala, M.D, Rosbeck, J.P., Lum, N.A., Lum, W.Y., Garnett, J.D., Hoffman, A.W., Domingo, G., Cushman, G.M, Rapchun., D.A., "InSb arrays for IRAC on SIRTF," SPIE Proc. 4131, pp. 7-12, 2000.

${ }^{10}$ McMurray, R., Johnson, R.R , McCreight, C.R., McKelvey, M.E., Garnett, J.D., Hoffman, A.W., Lum, N.A , Lum, W.Y.,. Smith, M.S., Sparkman, K.P., Toth, A.G., Domingo, G., Krebs, D. , Jhabvala., M. "Si:As Array Performance for SIRTF/IRAC," SPIE Proc. 4131, pp. 62-69, 2000.

${ }^{11}$ Ennico, K., McKelvey, M., McCreight, C., McMurray, R., Johnson, R., Love, P., Lum, N. "Large Format Si:As IBC Array Performance for NGST and Future IR Space Telescope Applications," these proceedings.

${ }^{12}$ McMurray, C.W., Forrest, W.J., Pipher J.L., "Next Generation Space Telescope: NIR InSb array development," these proceedings. 